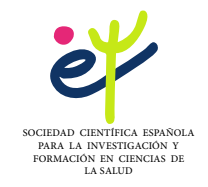

\title{
Taponamiento Cardiaco tras la reparación de una Hernia de Morgagni y de Hiato por Laparoscopía
}

\section{Cardiac Effusion after Repair of Morgagni and Hiato's Hernia by Laparoscopy}

\author{
Ricardo Muñoz García* \\ Hospital Regional Universitario de Málaga, España.
}

\section{RESUMEN}

La hernia de Morgagni es una malformación que se presenta en el 3-5\% de las hernias diafragmáticas, la mayoría son asintomáticas. La reparación mediante laparoscopia tiene varias ventajas sobre el tratamiento tradicional. La tasa de complicaciones está por debajo del $5 \%$. El taponamiento cardiaco es una rara complicación tras una cirugía laparoscópica.

Presentamos el caso de una mujer que tras ser intervenida de una hernia de Morgagni por laparoscopia sufre un taponamiento cardíaco. Se realizó un abordaje de urgencia, mediante esternotomía y se extrajo el dispositivo Tacker que provocó el derrame por laceración del ventrículo derecho a través del diafragma.

Palabras clave: hernia; tacker; taponamiento cardiaco; hernia de Morgagni

\begin{abstract}
Morgagni's hernia is a malformation that occurs in 3-5\% of diaphragmatic hernias, most of which are asymptomatic. Laparoscopic repair has several advantages over traditional treatment. The complication rate is below 5\%. Cardiac tamponade is a rare complication after laparoscopic surgery.

We present a case of a woman who, after undergoing laparoscopy for a Morgagni hernia, suffered cardiac tamponade. An emergency approach was performed, using a sternotomy, and the Tacker device that caused the effusion by laceration of the right ventricle through the diaphragm was removed.
\end{abstract}

Keywords: hernia; tacker; cardiac effusion; Morgagni hernia. 


\section{INTRODUCCIÓN}

La hernia de Morgagni es una malformación que se presenta en el 3-5\% de las hernias diafragmáticas (Terrosu et al., 2012), la mayoría son asintomáticas. La reparación mediante laparoscopía es una alternativa al tratamiento estándar, con bastantes ventajas sobre la cirugía abierta (Durak et al., 2007), que ha ido ganando adeptos desde su introducción en 1992. Las complicaciones que pueden aparecer mediante esta técnica están por debajo del 5\% (Horton et al., 2008).

Las hernias de hiato reparadas mediante técnicas laparoscópicas han demostrado ser factibles y seguras, a pesar de una mayor dificultad técnica, con resultados excelentes. El cierre de los pilares es uno de los pasos más dificultosos, y algunos autores recomiendan el uso de mallas para reforzar el cierre (Luostarinen et al., 1998).

Hay complicaciones severas relacionadas con la implantación de mallas en cirugía general, que están bien definidas, pero es más extraño el daño cardíaco en relación con el implante de estas mallas (Frantzides \& Welle, 2012).

El taponamiento cardíaco es una rara complicación tras la cirugía de reparación de hernias diafragmáticas. Puede aparecer por diversas etiologías, y producirse durante la cirugía abierta o mediante cirugía laparoscópica (Paz et al., 2011).

Presentamos el caso de una paciente intervenida de hernia de hiato y hernia de Morgagni, que tras la intervención laparoscópica sufre un taponamiento cardíaco.

\section{REPORTE DEL CASO}

Mujer de 74 años que tras ser intervenida de una hernia de hiato de gran tamaño, donde se encontró como hallazgo inesperado una hernia diafragmática de Morgagni, ingresó en la unidad de cuidados intensivos (UCI) a las 48 horas, tras sufrir malestar general con náuseas y síncope. En la cirugía laparoscópica se realizó, funduplicatura tipo floppy-Nissen y hernioplastia con malla bicapa de poliéster tridimensional con colágeno, en diafragma fijada por numerosos Tacker, tres de ellos metálicos.

En UCI, se realizó una tomografía axial computarizada (TAC), donde se evidenció derrame pericárdico moderado que ocupaba toda la cavidad pericárdica, con signos sugestivos de fracaso cardíaco y un impacto metálico, cerca de la superficie del corazón (imagen 1). 


\section{Imagen 1.}

TAC en el que se observa derrame pericárdico moderado y su relación con impacto metálico. En recuadro, tacker retirado.

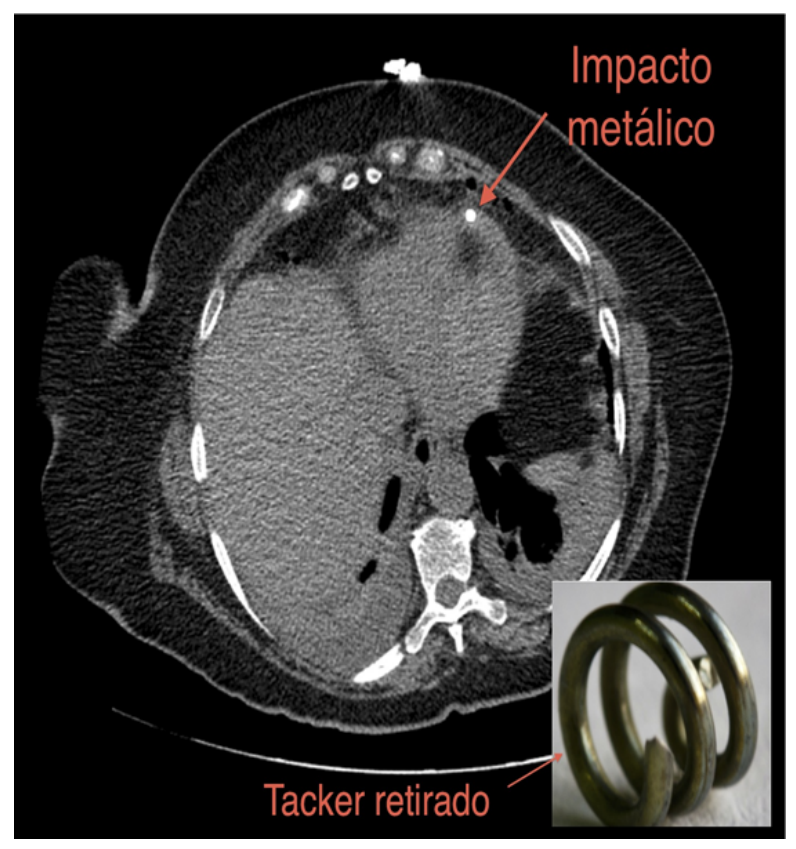

Se realizó una ecocardiografia y se observó un derrame pericárdico de cuantía moderadosevera, tabicado, con criterios ecocardiográficos de taponamiento cardiaco, por lo que, junto con la clínica de la paciente, se decidió cirugía urgente por esternotomía media con el fin de retirar el material que originaba el trauma miopericárdico, desestimándose el drenaje subxifoideo, intercostal o percutáneo.

Se intervino mediante esternotomía media, se realizó pericardectomía, evacuándose $800 \mathrm{ml}$ de sangre con coágulos y extrayéndose de la cara diafragmática un Tacker de $5 \mathrm{~mm}$ helicoidal de titanio (imagen 2), mediante disección con bisturí eléctrico e interposición de membrana de teflón y Surgicel ${ }^{\circledR}$ entre el corazón (el cual se encontró erosionado) y el diafragma. Se realizó cierre de esternón sin complicaciones intraoperatorias.

La paciente tuvo un postoperatorio sin incidencias, siendo dada de alta a los pocos días de la intervención y permaneciendo asintomática a los 12 meses de la misma. 


\section{Imagen 2.}

Fotografía del sitio quirúrgico de la pericardectomía con remoción del Tracker

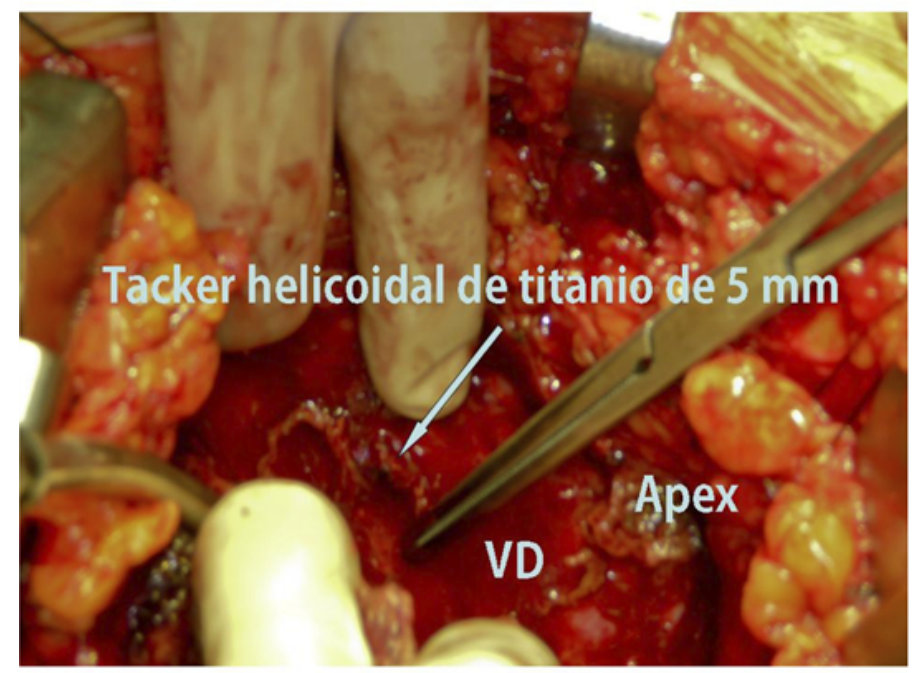

\section{DISCUSIÓN}

El taponamiento cardiaco tras la cirugía laparoscópica es una rara complicación que requiere drenaje urgente. Las manifestaciones clínicas del taponamiento post-laparoscopia pueden aparecer dentro de los 14 primeros días tras la cirugía (Thijssens et al., 2002).

El espesor del diafragma oscila entre $1.5 \mathrm{~mm}$ y $5.4 \mathrm{~mm}$, muchos de los tackers metálicos sobrepasarían esa distancia, por lo que podrían estar contraindicados en situaciones donde se podrían dańar tejidos en los cuales no se pudiese inspeccionar para una revisión de la hemostasia.

Actualmente existen diversos modelos tipo "tacker" de fijación por laparoscopia en el mercado, pudiéndose diferenciar esquemáticamente dos modelos: helicoidales no absorbibles de titanio y absorbibles. Éstos últimos ofrecen la ventaja de no dañar la superficie de órganos vitales en caso de entrar en contacto con ellos y, en caso de hacerlo, absorberse en un corto periodo de tiempo. También podemos encontrar un tipo de grapas que al cerrarse no sobrepasarían el espesor del diafragma, evitando así el daño a estructuras vecinas.

Aunque el taponamiento cardíaco es una rara complicación, debemos tenerla presente, ya que puede desencadenar en un trágico final. El implante de dispositivos en el tendón central del diafragma debería evitarse, y se debería considerar el uso de elementos que no 
sobrepasen el espesor del diafragma (sobretodo en zonas próximas al corazón) o dispositivos que se reabsorban con el paso del tiempo.

\section{REFERENCIAS}

Durak, E., Gur, S., Cokmez, A., Atahan, K., Zahtz, E., \& Tarcan, E. (2007). Laparoscopic repair of Morgagni hernia. Hernia : the journal of hernias and abdominal wall surgery, 11(3), 265-270. https://doi.org/10.1007/s10029-006-0178-z

Frantzides, C. T., \& Welle, S. N. (2012). Cardiac tamponade as a life-threatening complication in hernia repair. Surgery, 152(1), 133-135. https://doi.org/10.1016/j. surg.2011.08.009

Horton, J. D., Hofmann, L. J., \& Hetz, S. P. (2008). Presentation and management of Morgagni hernias in adults: a review of 298 cases. Surgical endoscopy, 22(6), 14131420. https://doi.org/10.1007/s00464-008-9754-x

Luostarinen, M., Rantalainen, M., Helve, O., Reinikainen, P., \& Isolauri, J. (1998). Late results of paraoesophageal hiatus hernia repair with fundoplication. The British journal of surgery, 85(2), 272-275. https://doi.org/10.1046/j.13652168.1998.00508.x

Paz, Y. E., Vazquez, J., \& Bessler, M. (2011). Cardiac tamponade as a complication of laparoscopic hiatal hernia repair: case report and literature review. Catheterization and cardiovascular interventions: official journal of the Society for Cardiac Angiography \& Interventions, 78(5), 819-821. https://doi.org/10.1002/ccd.23178

Terrosu, G., Brizzolari, M., Intini, S., Cattin, F., Bresadola, V., \& De Anna, D. (2012). Morgagni hernia: technical variation in the laparoscopic treatment. Annali italiani di chirurgia, 83(5), 415-420. https://pubmed.ncbi.nlm.nih.gov/22615038/

Thijssens, K., Hoff, C., \& Meyerink, J. (2002). Tackers on the diaphragm. Lancet (London, England), 360(9345), 1586. https://doi.org/10.1016/S0140-6736(02)11530-3

Recibido: 27 de diciembre de 2020

Aceptado: 15 de febrero de 2021 